\title{
Coulisses
}

Revue de théâtre

\section{Mesure pour mesure de William Shakespeare}

Mise en scène de Jean-Yves Ruff

\section{Claude Peltrault}

\section{CpenEdition}

Journals

Édition électronique

URL : https://journals.openedition.org/coulisses/949

DOI : 10.4000/coulisses.949

ISSN : 2546-9460

Éditeur

Presses universitaires de Franche-Comté

\section{Édition imprimée}

Date de publication : 31 décembre 2009

Pagination : 13-17

ISBN : 978-2-84867-270-0

ISSN : $1150-594 \mathrm{X}$

\section{Référence électronique}

Claude Peltrault, « Mesure pour mesure de William Shakespeare », Coulisses [En ligne], 39 | Automne 2009, mis en ligne le 30 novembre 2016, consulté le 29 décembre 2022. URL : http://

journals.openedition.org/coulisses/949; DOI : https://doi.org/10.4000/coulisses.949

Ce document a été généré automatiquement le 29 décembre 2022.

Tous droits réservés 


\section{Mesure pour mesure de William Shakespeare}

Mise en scène de Jean-Yves Ruff

Claude Peltrault

\section{RÉFÉRENCE}

Mesure pour mesure de William Shakespeare

Mise en scène de Jean-Yves Ruff. Coproduction : Théâtre Vidy-Lausanne, MC93 Bobigny, Chat Borgne Théâtre, Le Maillon-Théâtre de Strasbourg.

Spectacle vu le jeudi 11 décembre 2008 au Théâtre musical de Besançon.

1 La recherche d'un juste et équitable exercice du pouvoir est au centre de cette œuvre classée parmi les pièces problématiques de Shakespeare. Le Duc de Vienne prend soudain conscience d'avoir trop peu et mal exercé son autorité depuis quatorze ans et décrit l'état du pays comme un monde à l'envers en reprenant l'imagerie populaire de l'époque : le bébé corrige la nourrice, etc. Pour ne pas tomber d'un excès de laxisme dans la tyrannie, il décide de prendre du champ et de déléguer son pouvoir à Angelo. En réalité ce départ est une feinte, le Duc veut surtout se livrer, dans le plus grand secret, à une expérience de laboratoire et, déguisé en moine, observer si le pouvoir va ou non infléchir la rectitude puritaine d'Angelo, et peut-être faire voler en éclats sa vertu qui ne serait que de façade. La dialectique de l'ombre et de la lumière, du caché et du montré, la duplicité aussi, sont très bien suggérés dès la première minute, par la mise en scène de Jean-Yves Ruff. Le Duc n'est d'abord qu'une voix qui monte des ténèbres : il est totalement invisible quand il informe de son projet le magistrat Escalus, planté dans le faisceau d'un projecteur où il est rejoint par Angelo. La scène suivante révèle à travers la semi transparence d'un rideau de voile noir, l'intérieur d'une maison de plaisir, baigné d'une lumière rouge dans laquelle s'activent des ribaudes très dévêtues, autour et dans un grand bassin peu profond qui, vidé après la scène du couvent, occupe une grande partie de la scène et sert ainsi de trait d'union critique entre les différents 
lieux de l'action, les hautes sphères de la justice et les bas-fonds, le mélodrame et la farce.

Multifonctions, ce bassin est d'abord le vivier dans lequel patauge la faune du bordel, y compris Madame Foutue, la mère maquerelle haute en couleurs dont Laurent Menoret, qui lui prête sa stature de rugbyman, fait une savoureuse caricature. Jouxté par une fontaine destinée à la toilette, le bassin sert aussi de pissotière au tavernier bouffon, Pompée, et à sa clientèle, puis un humour de potache en fait un bénitier quand l'action se transporte au couvent d'Isabelle. L'intimité du bordel ainsi entrevue par le spectateur le transforme en voyeur et lui impose de partager la curiosité quelque peu malsaine du Duc. En même temps se trouve bien posée dès cette deuxième scène, avec l'ambivalence du rideau qui dévoile autant qu'il voile, avec la maquerelle représentée par un travesti, la dangereuse et souvent comique promiscuité des contraires. Le zélé Ducoude (Xavier Legrand), un de ces agents de police peu regardants sur le langage dont Shakespeare adore se moquer, se vante d'arrêter des « bienfaiteurs", tandis que le vice s'épanouit chez le mal nommé Angelo.

Dans la brillante scénographie de Laure Pichat il faut aussi souligner l'efficacité du dispositif de fond de scène qui permet d'insérer de manière très propre les scènes d'observation ou d'aparté. Il s'agit d'un large panneau dans lequel s'ouvrent et se ferment, par un système de coulisses, des espaces suggérant les cellules de la prison, ou la cachette d'où le Duc peut épier une scène ou avoir une entrevue secrète, comme lorsqu'il organise sa double vie avec la complicité d'un moine. A ce moment, l'éclairage (Stéphanie Petton) transforme la surface du bassin en miroir et souligne la duplicité du personnage qui s'y trouve réfléchi. Autre trouvaille en forme de clin d'oeil, dans la moitié supérieure gauche de ce panneau, se niche une curiosité horlogère: un automate représentant peut-être un avatar de l'aigle autrichien ou un grand-duc, ce qui revient au même, rythme l'action de ses battements d'ailes métalliques, comme le Duc d'Autriche la dirige, et souvent de façon mécanique, avec ses manipulations.

Quelques mots maintenant sur l'interprétation des personnages, et ce qu'elle nous dit de la vision du metteur en scène. Mesure pour mesure est une pièce problématique, pas seulement en raison du mélange des genres, fréquent dans l'œuvre de Shakespeare, mais parce que les germes tragiques s'y trouvent en quelque sorte génétiquement installés. Environ huit ans plus tôt, Shakespeare avait écrit sa deuxième tragédie, Roméo et Juliette. Il se trouve que le Duc reconnaît avoir négligé sa tâche pendant 14 ans, c'està-dire l'âge qu'a Juliette lorsqu'elle rencontre Roméo. Dans Mesure pour mesure, Juliette porte l'enfant illégitime de Claudio, faute d'avoir pu l'épouser, pour des raisons financières qui ont tout à voir avec l'entente des familles, et l'un des magistrats auxquels le Duc confie le pouvoir, se nomme Escalus, comme le gouverneur de Vérone. En plus de ces réminiscences qui hantent l'inconscient du texte, il y a dans Mesure pour mesure un réalisme macabre, très développé aussi dans Roméo et Juliette, et ici mis au service d'une farce assez grimaçante. Jean-Yves Ruff nous montre un bourreau menaçant (Laurent Menoret), qui aiguise sa hache en produisant des gerbes d'étincelles, pendant que Claudio (Igor Mendjisky, faible et pitoyable à souhait) tremble face à la mort. Angelo exige qu'on lui apporte la tête de Claudio, auquel le Duc doit trouver un suppléant, ce qui débouche sur la comédie grotesque du condamné à mort récalcitrant (Jacques Tresse), que Pompée, passé tout naturellement des bas-fonds aux hautes œuvres, tente vainement de convaincre. Dans son rôle de bouffon un peu ivrogne, un peu maquereau, Alexandre Soulié campe un personnage pittoresque: grand 
et gras, ventre à l'air, culotte fendue derrière, longs cheveux nattés, moustache tombante et bouc, tout chez lui exprime le débordement. L'acteur se laisse même emporter à déborder de son texte, car ce 11 décembre 2008, inspiré par la dernière polémique autour du Secrétariat aux Droits de l'Homme, il s'autorise à citer le nom de Rama Yade!

Ce qui fait la singularité de cette comédie, qui se termine, selon un respect mécanique de la tradition, par de multiples mariages, c'est aussi l'absence de romantisme et l'impression de malaise qui demeure après un dénouement infiniment retardé. Il en résulte un questionnement persistant qui ouvre à tout metteur en scène un vaste espace d'interprétation. La vision personnelle de Jean-Yves Ruff s'exprime notamment à travers sa direction de Laetitia Dosch et Jérôme Derre.

Incarné par ce dernier, le personnage du Duc évolue vers une sorte de psychopathe, ou à tout le moins quelqu'un de très inquiétant. Ce n'est pas par hasard que l'antagoniste comique de ce personnage de l'ombre se nomme Lucio (Pierre Hiessler, culotté et couard avec talent). La duplicité et l'art de la dissimulation du Duc, égaux à ceux d'Angelo, sont bien soulignés par l'éclairage. Pendant le soliloque: "Que cache l'homme sous les traits de l'ange...", une lumière crue lui illumine une moitié du visage, pendant que l'autre est perdue dans les ténèbres. On voit son excitation et sa jubilation monter au fur et à mesure que s'enrichit son intrigue, dont il retarde cruellement le dénouement en inventant toujours de nouvelles manipulations. Lorsque Isabelle s'apprête à quitter la cellule de Claudio, outrée de voir son frère lui demander de sacrifier sa virginité pour le sauver, le Duc l'arrête avec de grandes gesticulations de bras, mouvements compulsifs qui tendent à se répéter et font parfois penser au jeu hypernerveux d'un De Funès. Lui qui dit au premier acte avoir répugné à exercer pleinement l'autorité de son magistère, nous le voyons s'amuser comme un fou à faire lever et rasseoir un Prévôt tremblant de peur (Jacques Hadjaje) plusieurs fois de suite sur un mot, comme un enfant qui découvre les marionnettes. Jérôme Derre ajoute à cela un timbre de voix singulier, avec des vibrations un peu inquiétantes, d'étranges contorsions, et une fluidité de couleuvre assez en rapport, après tout, avec la fragilité de la frontière entre le bien et le mal dans la pièce. En tout cas, si le Duc, sous son déguisement, est un double de l'auteur/acteur/metteur en scène, la composition ne manque pas d'ironie.

7 Quant à Isabelle, interprétée par Laetitia Dosch, elle nous apparaît d'abord tête et pieds nus, seulement vêtue d'une chemise immaculée, qui évoque l'habit des Clarisses, mais pourrait tout aussi bien être la chemise de nuit d'une somnambule. Dans le ton de sa voix la niaiserie l'emporte sur la naïveté, ce qui rend tout d'abord ce personnage de petite oie blanche inintéressant, et inapte à susciter l'amour. Des rires s'élèvent dans le public lorsqu'elle débite maladroitement sa prière pour fléchir Angelo. Mais on peut comprendre le metteur en scène s'il pense nécessaire cette dévaluation d'Isabelle pour traduire la nature du désir d'Angelo, car celui-ci n'est pas charmé par la femme, mais tenté de désacraliser la future religieuse. Toutefois, lorsqu'elle lui demande de s'interroger sur sa propre faiblesse, le ton change, la voix se fait plus basse, s'incarne plus chaudement pour subjuguer un Angelo tétanisé. Après son départ, celui-ci constate la nature perverse de son désir, dans une lumière rouge qui rappelle la scène du bordel et souligne la continuité entre le vice et la vertu supposés. Eric Ruff, ange blond revêtu de la stricte livrée noire du puritain et du démon, joue son personnage avec nuances mais sans se départir d'une froide retenue. C'est le feu sous la glace, 
excellente interprétation de la proximité des contraires. Quand Isabelle revient à la charge, le lendemain, et qu'Angelo prend symboliquement possession de son corps, une main sur sa tête, l'autre bras étreignant son épaule, elle retrouve ses réactions de petite sotte et reste hébétée, la bouche grande ouverte. Le contraste est total avec Noémie Dujardin, dont l'interprétation fait de Marianne, autrefois fiancée à Angelo, un personnage à la sensualité nettement plus éveillée. Comme pour le bouffon, mais avec moins de justification, l'excès tire le personnage vers le grotesque. C'est couchée de tout son long, et miaulant à la lune sa frustration, qu'elle écoute la chanson évocatrice qui s'égrène en coulisses: "Rendez-moi le désir/ Rendez-moi mon amour... », juste avant de recevoir la visite du faux moine qui va exaucer sa prière. Il est vrai que dans la pièce le grotesque n'est jamais bien loin, car c'est par des tours de passe-passe dignes du jeu de bonneteau pratiqué à la taverne que la tête de Ragozin est amenée à Angelo pour conserver à sa place celle de Claudio, et que la virginité de Marianne est offerte dans l'ombre à Angelo pour préserver celle d'Isabelle. Pas pour longtemps : le Duc lui propose le mariage de façon assez péremptoire et, sans attendre de réponse, quitte la scène avec sa suite. Isabelle reste seule, muette, de dos et comme hébétée, au milieu du bassin dans lequel l'eau se remet à couler, diluant un liquide rouge contenu dans une ampoule, possible allusion à la défloration et retour aux eaux rougies de la scène du bordel.

8 Quelque conclusion que l'on tire de tout cela, il y a en tout cas de la part de Jean-Yves Ruff une vision personnelle et stimulante d'une œuvre complexe dont on n'oubliera pas de mentionner l'habile traduction d'André Markowicz.

\section{Mesure pour mesure, photographie de scène}

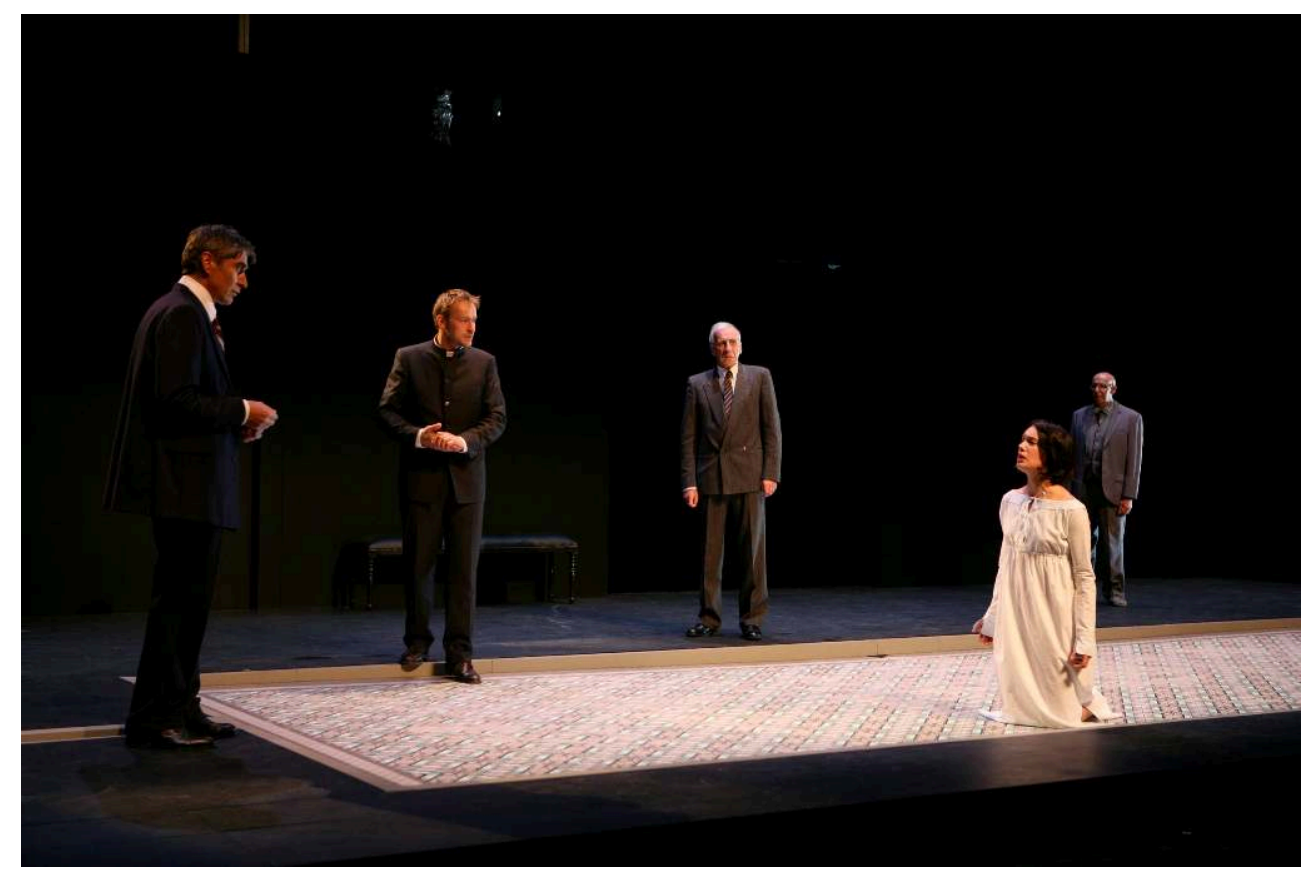

Photo Mario Del Curto 